\title{
Qos Constrained Communication using Voice Codecs over Multicast Ad Hoc Networks
}

\author{
Amit Chopra \\ PhD Research Scholar, \\ CSE Deptt., MMEC, \\ M. M. University, Ambala, India
}

\author{
Rajneesh Kumar, PhD \\ Professor \\ CSE Deptt., MMEC, \\ M. M. University, Ambala, India
}

\begin{abstract}
QoS support for group communication over MANETs using real time applications is essential to fulfill the requirements of users. It is very challenging for a multicast ad hoc routing protocol to perform under the QoS constraints due to the unstable network environment, unpredictable traffic loads, mobility, scalability and limited resources etc. Researchers have developed some standards to support real time audio/video traffic over network such as Voice over IP (VoIP), H.323, H.264 etc. Multicast ad hoc routing protocols are designed to work with the traditional traffic streams, so there is need to analyze their performance using real time traffic in order to extend their capabilities. In this paper, we will explore the efficiency of PUMA multicast routing protocol using various voice codecs under the QoS constraints.
\end{abstract}

\section{Keywords}

Multicast, QoS, MANETs, PUMA, VoIP, Voice Codec

\section{INTRODUCTION}

Multimedia applications contain audio and video data packets which are transmitted over network with minimum time delay can be referred as called real time applications. Audio data contains only the voice frames where as video data may contain both audio and video frames. This type of data transmission should fulfill the QoS constraints otherwise end users will not be able to utilize the applications properly. Multimedia traffic suffers from various factors like node movements,jitter, end to end delay, behavior of routingprotocol, hardwareconfiguration ofsender/receiver/intermediate devices, bit error rate, restricted bandwidth and radio area coverage etc. [1]

\subsection{Audio/Video Streams and libraries}

Audio/Video transmission over MANETs can extend the capabilities of the real time applications and users can establish a group based network. In multicast communication, Sender or receiver, any one can initiate the connection. Researchers have developed many solutions for real time multimedia applications to fulfill the QoS requirements. These applications utilize some standard libraries like H.323 and H.264 etc. Although these libraries were developed to support the multimedia applications over internet but we can also use them over ad hoc networks. Now we will discuss about them in next section [2].

\subsection{Voice over IP}

VoIP standard was developed to establish voice communication over internet. Users can communicate in real environment but quality of communication depends upon the availability of the bandwidth and the concurrent users. Voip based applications work with other protocols such as Gateway, Transport and Signaling protocols. These group of protocols perform different operations including signal conversion, session establishment, Call control, address resolution and optimization of resource utilization [1][3]. H.323 was developed for VoIP communication over different networks to support the large traffic volume [4].

\subsection{H.323}

International Telecommunication union (ITU-T) has defined the standards for real time end to end/multipoint/broadcast communication support over networks which are as follow [5]:

Table:1 ITU-T Standards [5]

\begin{tabular}{|c|c|}
\hline Standard(s) & $\begin{array}{c}\text { Use } \\
\text { H.10-H.19 } \\
\text { telephony }\end{array}$ \\
\hline H.20-H.29 & $\begin{array}{c}\text { Channels used for other than } \\
\text { Voice Frequency Telegraphy Circuits }\end{array}$ \\
\hline H.30-H.39 & $\begin{array}{c}\text { Telephone Cables/Circuits for } \\
\text { Telegraph }\end{array}$ \\
\hline H.40-H.49 & Fax/Telegraphy Circuits \\
\hline H.50-H.99 & Signaling \\
\hline H.100-H.199 & Audiovisual Infrastructure \\
\hline H.200-H.219 & General \\
\hline H.220-H.229 & Synchronization/Multiplexing \\
\hline H.230-H.239 & System Aspects \\
\hline H.240-H.259 & Methods used for Communication \\
\hline H.260-H.279 & Moving video Coding \\
\hline H.280-H.299 & Related systems \\
\hline H.300-H.399 & Devices for Audio/Video Services \\
\hline H.450-H.499 & Multimedia's optional services \\
\hline
\end{tabular}

H.323 includes the various components given below:

1. Gate Keepers: Resource management

2. Gateways: Communication between other networks

3. Terminals/Multipoint Control Units: for end users

4. Border/Peer Elements: Optional

5. Signaling Method: SIP/RSA/H.245

6. Codec: G.711, G.729, G.729a, G.723.1, G.726 [6][17]

Voice codecs can be categorized on the basis of data rate, sampling rate, signaling and compression techniques etc. [9]

Table:2 Voice Codecs [9][12]

\begin{tabular}{|c|c|c|c|}
\hline Voice Codec & Bit rate & Interval & Payload \\
\hline G.711 & 80 & 80 & 160 \\
\hline G.729a & 8 & 30 & 20 \\
\hline G.723.1 & 6.3 & 24 & 33 \\
\hline GSM & 13.2 & 20 & 33 \\
\hline
\end{tabular}

\subsection{PUMA Multicast Routing Protocol}

PUMA is a multicast routing protocol in which receiver initiates the connection and sender can communicate with the 
group without becoming its member. It offers high PDR and can also reduce the control overhead in order to enhance the network performance. It uses announcements for mesh management and packet flow is restricted with in the mesh only but in long term this is not suitable for large traffic volume [8]. PUMA protocol performs following network operations:

a. Mesh Establishment: Mesh is built on the basis of receiver using shortest path along with a core which is elected using election method that regulates announcement packets within the mesh.

b. Mesh Maintenance: Routing information is maintained by each node on the basis of received announcement packet.

c. Core Node Selection: Core Node is elected on the basis of highest Node id and core election methods.

d. Data Packet Forwarding: Packets can be sent to core in unicast manner and later on flooding is used within the mesh.

e. Multicast Announcement Propagation: Single Control Packet is used for network operations to reduce the overhead and it contains Id of Core node, group, members,parent, sequence number, core distance, next hope etc. Multicast Group list uses core dependent and independent parameters i.e. last received announcement packet, sequence number, core id and connectivity list depend upon core and multicast addressing, flags related to mesh member and receivers are independent from Core [7].

\section{LITERATURE REVIEW}

Application domain of MANET is increasing day by day. Researchers have developed many solutions to provide the QoS support for real time applications over MANET using traditional communication protocols. Now will discuss the efforts made by them. V.Srihari et al. [1] did a survey of the security threats and their remedies for VoIP/SIP protocols. As per their studies, security attacks can be introduced on session management, signaling, call control and credits etc. For detection and prevention, service providers can use intrusion detection system, fake call monitoring system, call analysis and pattern recognition etc.

Guoping Tan et al. [8] developed a solution for real time communication over mobile ad hoc networks, called Partial Network Coding based Real-time Multicast. It is capable to reduce the delay while maintaining the throughput under the dynamic environment. Each node performs some specific operations such as encoding, forwarding and decoding etc. These are performed by sender, receiver and intermediate nodes in the mesh network which operates on the concept introduced by a another multicast protocol, called PUMA protocol. Sender produces a stream of packets having same size with unique identification numbers. At the time of packet reception, agent node arranges them in to number of unique blocks as per packet sequence number and assign a block id to each block. Intermediate nodes maintain a buffer with a matrix. Buffer status can be altered as the rank of matrix. For forwarding purpose, re-encoding of the buffered packets is done to minimize the delay. Receiver accepts the incoming packets and differentiates them in to parent/child using block size and updates the buffer status. Simulation results show that it can adopt the variations in receiver's density and can perform well in mobile environment.

Pratyush Manjul et al. [9] examined the performance of different ad hoc routing protocols by establishing multicast communication using video steams. They considered the various performance parameters like throughput, packet loss and latency etc. Simulation results show that OLSR routing protocol can perform better than the PUMA protocol which suffers from the packet loss, node density and congestion at sender side.

M. Iqbal et al. [10] developed a solution for multimedia services using Linux platform, called unidirectional linkaware MAODV which is based on AODV-UU protocol. In order to reduce extra control overhead, it uses only reliable links for route discovery and UDP protocol for real time load which increases transmission reliability. Authors used different platforms to review the performance of proposed solution and Simulation results show its efficiency under the QoS constraints i.e. bandwidth and delay etc.

Minho Park et al. [11] presented a call admission control method for VoIP based on IEEE 802.16 standard. It utilizes the resources of the network in such a way that free bandwidth can be used by different nodes at same time and estimation for bandwidth assignment to each node is based on the multiplexing gain and probabilistic schemes and by subdivided the voice streams using two state markovchain. It can set priorities of hanoff and new calls on the basis of acceptance ratio. Simulation results show its effectiveness and efficiency in terms of resource utilization and management.

Ritesh K. Kalle et al. [12] proposed a energy efficient method for VoIP communication over IEEE-802.16m based networks by reducing overheads. During the data transmission phase, mobile station remains active and uses packet buffering. As per their analytical analysis, they categorized the power consumption in different modes i.e. idle mode, transmission mode and receiving mode and finally they examined the cycles in each mode. Simulation results show that power can be preserved in sleep mode by optimizing the MAC layer, by reducing overhead and delay etc.

Saran Chiwtanasuntorn et al. [13] proposed a Voice over IP scheme which can utilize multiple TCP connections. Although TCP is not suitable for multimedia applications due to delay, jitter and higher packet loss ratio etc but they presented a VoMTCP method which can send the VoIP packets using multiple TCP connections by arranging audio stream in to coding units defined by various codec standards (G.711/G.729). At receiver end, received data is finally decoded. Simulation results show that variation in number of TCP connections, queue length, network density and transmission parameters affect the voice quality. By selecting of the feasible values of all these parameters, Packet Loss Ratio can be controlled but authors did not consider the other parameters i.e. end to end delay and jitter etc. which are quite important for measuring QoS constraints [8].

Yi Deng et al. [14] did analysis of voice transmission over ad hoc networks established in underground mines using different voice codecs i.e. G.711, G.723, and G.729 etc. They used two state error model with AODV routing protocol and vary the VoIP sessions from 5 to 10 with the node mobility $3 \mathrm{~m} / \mathrm{s}$. Simulation results show that G.723 has better performance in terms of Mean Opinion Score and offers minimum packet loss and delay as compared to G.729 and G.711 is not suitable. Results also show that quality of VoIP is affected due to obstacles in underground mines

Zahraa Sabra et al. [15] proposed a end user solution which is capable to provide secure environment for VoIP communication with respect to QoS parameters using hybrid ad hoc networks. They used AES, ECC192 to implement 
security features and Voice codec G.729b for simulation purpose. As per the proposed scheme,

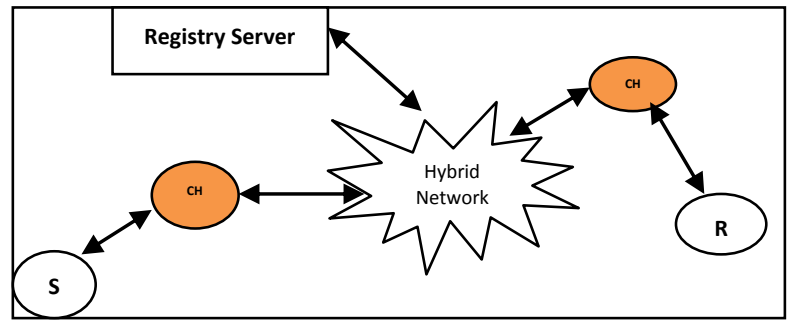

Fig 1: Hybrid Network [15]

Sender and receiver communicate through cluster heads which are randomly selected and these are connected to hybrid networks. Shared keys are computed using SHA-512. Sender side cluster head sends query to Registry server which is forwarded to receiver side cluster head. If cluster heads share same keys, only then a link is established on the basis of authentication, after that sender and receiver can start VoIP communication. If a new cluster head is selected then registry server updates this information. Simulation results show that this scheme offers authentication against non-repudiation and traceability under QoS constraints.

Ramon Sanchez-Iborra et al. [16] did analysis of ad hoc routing protocols under different constraints i.e. fading characterization, node density and node mobility etc. For simulation purpose, they used Nakagami-m propagation model, OLSR and BATMAN routing protocols, node density varies from 25 to 50 nodes, space vary from $50 \mathrm{mx} 50 \mathrm{~m}$ to $125 \mathrm{mx} 125 \mathrm{~m}$ and velocity based on Gaussian distribution method. Simulation results show that in case of channel gadding, BATMAN performs better than OLSR but in case of other factors, OLSR performance is better.

\section{SIMULATION AND RESULTS ANALYSIS}

Table 3. Simulation Scenario

\begin{tabular}{|c|c|}
\hline \multicolumn{2}{|c|}{ Simulation Scenario } \\
\hline Nodes & 30 \\
\hline Sender & $1,5,10,15$ \\
\hline MAC & 802.11 \\
\hline Terrain & $1200 \times 1200$ \\
\hline $\begin{array}{c}\text { Ad Hoc Multicast } \\
\text { Routing Protocol }\end{array}$ & PUMA \\
\hline Simulation Time & 600 Seconds \\
\hline Group Size & 1 \\
\hline Propagation Model & TwoRayGround \\
\hline Simulator & NS-2 \\
\hline
\end{tabular}

Table4. Sender vs Receivers

\begin{tabular}{|c|c|}
\hline \multicolumn{2}{|c|}{ Sender vs Receivers } \\
\hline $\begin{array}{l}\text { Number of } \\
\text { Sender(s) }\end{array}$ & Number of Possible Receiver(s) \\
\hline 1 & 29 \\
\hline 5 & 25 \\
\hline 10 & 20 \\
\hline 15 & 15 \\
\hline
\end{tabular}

Table5. VoIP Configuration

\begin{tabular}{|c|c|}
\hline \multicolumn{2}{|c|}{ VoIP Configuration } \\
\hline MOS Threshold & 3.0 \\
\hline Voice Codecs & $\begin{array}{c}\text { G.711, G.729A, G.723.1, } \\
\text { GSM.AMR, GSM.EFR }\end{array}$ \\
\hline Exponential Talk & 1 \\
\hline Exponential Silence & 1.5 \\
\hline Loss Threshold & 0.02 \\
\hline Frames per Packet & 2 \\
\hline
\end{tabular}

We used NS-2 network simulator to analyze the performance of multicast routing protocol using different voice codec. Following Table: shows the simulation scenario. We used Various voice codecs [6][18], PUMA multicast routing protocol, MAC 802.11 and sender vary from 1 to 15 and total nodes are 30.Table: 3 shows the basic simulation scenario.

Table:4 shows the number of sender vs number of possible receivers and Table:5 shows VoIP configuration used in simulation.

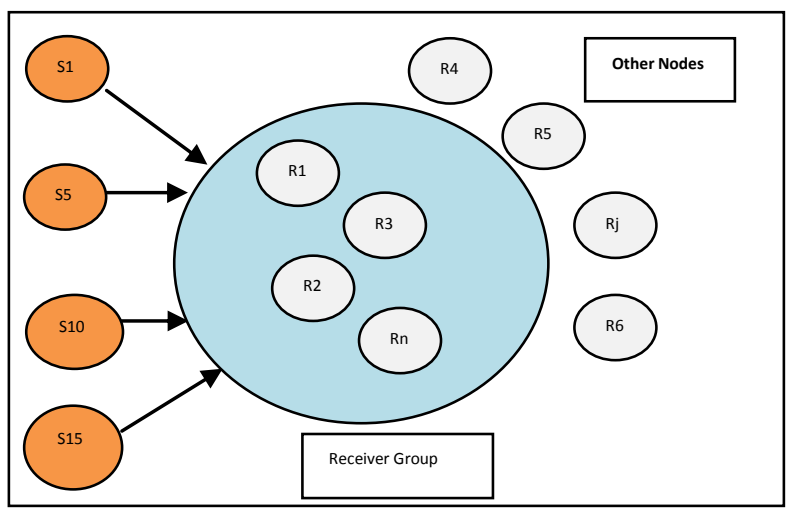

Fig 2: Multicast Communication

Fig 2: above shows the number of possible senders which can vary from 1 to 15 only and number of receivers can be vary upto 29 nodes that means at least one sender/receiver should be there for communication. Nodes can join and leave the group at any time. We can also draw an equation given below:

Total Nodes: $=\mathrm{nn}=30$

Sender: $=\mathrm{Si}=\{1,5,10,15\}$

Number of possible Receiver: $=\mathrm{Rn}=\left(\sum \mathrm{nn}-\sum \mathrm{Si}\right)$

Receiver: $=\mathrm{Rn}=\{29,25,20,15\}$ w.r.t sender(s)

Where $\mathrm{Si} \neq$ Null, $\mathrm{R} \neq \neq$ Null

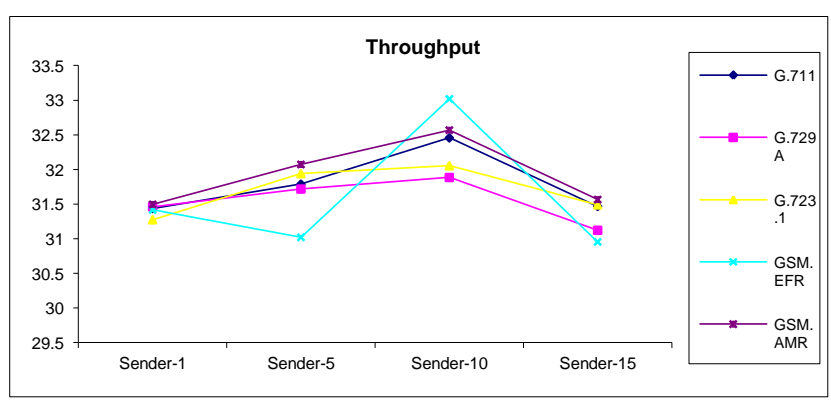

Fig 3: Throughput

Fig 3: below shows the Throughput of the network. As the number of sender increasing, Throughput is also increasing proportionally but in case of 15 senders, it isdecreasing 
rapidly till the end of simulation. If there is only one sender, Throughput is all most same with little bit variation, using all voice codecs.

In case of 5 and 10 senders, it is increasing and with 15 senders, it is decreasing. With 5 senders using GSM.AMR, it is highest and using GSM.EFR, it is lowest. Using G.729A and G.723.1, it is at medium level. If there are 15 senders then it is decreasing, using all voice codecs.

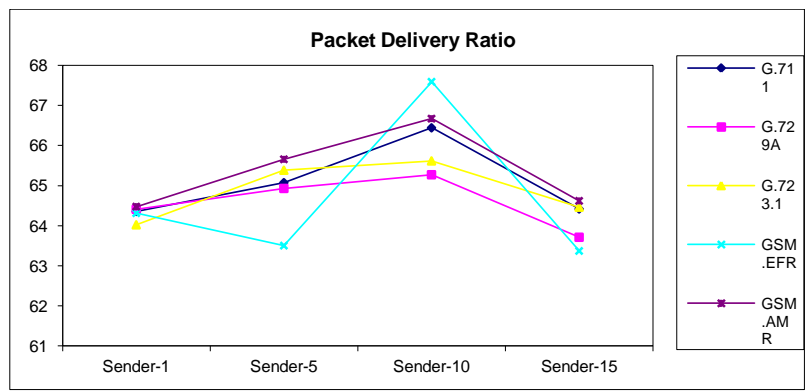

Fig 4: Packet Delivery Ratio

Fig 4: above shows PDR, using various voice codecs with the sender(s) $1,5,10,15$. If there is only one sender, PDR is stable, using all voice codecs. In case of 5 and 10 senders, there is variation in PDR and with 15 senders, it is decreasing in continues manner till the end of simulation.

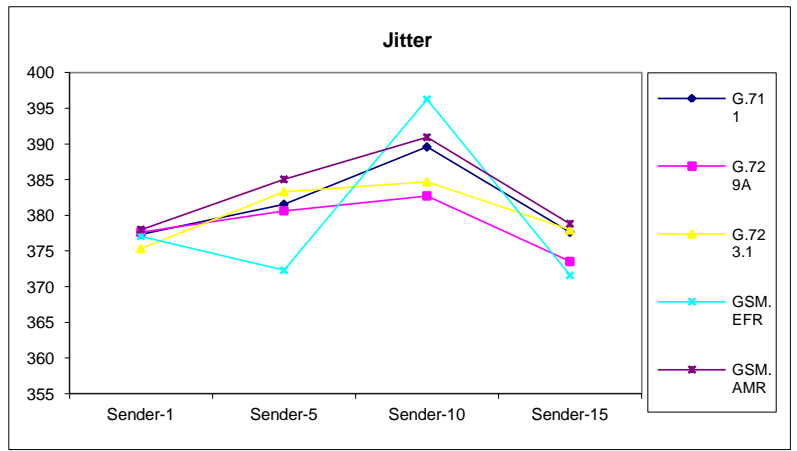

Fig 5: Jitter

Fig 5: shows the variations in jitter with respect to sender density. In case of sender(s) 1,15 , it is lowest and with 5 senders, it is at medium level and with 10 senders, it is highest level.

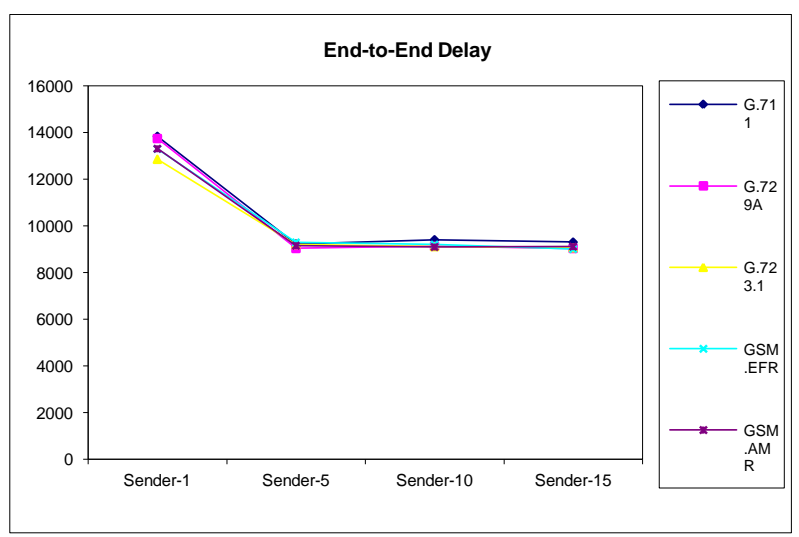

Fig 6: End-to-End Delay

Fig 6: above shows the variations in end-to-end delay. In case of one sender, It is highest and with sender 5, 10, 15, there is little bit variation.

\section{CONCLUSION}

In this paper, we examined the performance of PUMA routing protocol using different voice codecs. Simulation results show that variation in sender density nodes in a group can affect the performance of routing protocol. If number of sender increases, Throughput is also increasing proportionally but in case of 15 senders, it is decreasing rapidly till the end of simulation. If there is only one sender, Throughput is maintained with small variation, using all voice codecs, with 5 and 10 senders, it is increasing but with 15 senders, there is degradation in Throughput.. With 5 senders using GSM.AMR, it is highest and using GSM.EFR, it is lowest. Using G.729A and G.723.1, it is at medium level but with 15 senders, it is decreasing, using all voice codecs. In case of PDR, If there is only one sender, PDR is stable, using all voice codecs. In case of sender density 5 and 10, results show variation in PDR and with 15 senders, it is decreasing in continuesmanner till the end of simulation. Jitter is lowest with sender(s) 1,15 and with 5 senders, it is at medium level and with 10 senders, it is highest level. As per results, End-toEnd delay is highest with 1 sender and with senders 5, 10, 15, there are little bit variations. Finally we can conclude that if there is only 1 sender, then Throughput is at medium level, jitter is low and End-to-End delay is highest but when senders are 5 or 10, Throughput, jitter and PDR are increasing with the variations in delay. In case of 15 senders, there is degradation in all these parameters except End-to-End delay which is maintained at medium level till the end of simulation. In future, we will analyze the performance of ad hoc multicast routing protocols using video streaming.

\section{REFERENCES}

[1] V.Srihari, P.Kalpana, "Security Aspects of SIP based VoIP Networks: ASurvey", ICCTET, IEEE-2014, pp$143-150$

[2] MazinAlshamrani, Haitham Cruickshank, Zhili Sun, VahidFami, and Basil Elmasri"Evaluation of SIP Signalling and QoS for VoIP Over MANETs Reactive Routing Protocols",Next Generation Mobile Apps, Services and Technologies, IEEE-2013, pp-105-110

[3] Weiwei Zhang1, Yongyu Chang1, Yitong Liu1, Yuan Tian2, "Perceived QoS Assessment for Voip Networks", ICCT-2013, pp-707-711

[4] Twisa Mehta, ZunnunNarmawala, "Performance Enhancement of Multimedia Traffic over Wireless Ad hoc Networks using Network Coding",NUiCONE-2012, pp-1-6

[5] http://www.cisco.com

[6] http://en.wikipedia.org/wiki/H.323

[7] A.MenakaPushpa, K.Kathiravan, "Resilient PUMA (Protocol for Unified Multicasting through Announcement) against Internal Attacks in Mobile Ad Hoc Networks", ICACCI, IEEE-2013, pp 1906-1912

[8] Guoping Tan, Betene A. Francis Lin, Xinhua Peng,Xiuquan Liu, Chuanyu Qu, "A Partial Network Coding Based Real-Time Multicast Scheme In Manets", IETICT, IEEE-2013, pp-370 - 375

[9] PratyushManjul, VimaladhithanBalasubramanian, Yunzhi Li, Yuqing Liu, Yuan Shi, Jing Xu, "Real-time Video Streaming Over Multi-hop Ad-hoc Networks", IInd International Conference on Networking and Distributed Computing, IEEE-2011, pp 283 - 288 
[10] M. Iqbal, X. Wang D. Wertheim, "Reliable multimedia multicast communications over wireless mesh networks", IET Communication, 2010, Vol. 4 (11), pp. 1288-1299

[11] Minho Park, Dong Kun Noh, and Souhwan Jung, "An Efficient Uplink Admission Control for ertPS in IEEE 802.16", ICOIN, IEEE-2014, pp 395-400

[12] Ritesh K. Kalle, Maruti Gupta, Aran Bergman, EladLevy,ShantidevMohanty,Muthaiah Venkatachalam, Debabrata Das, "Advanced Mechanisms for Sleep Mode Optimization of VoIP Traffic over IEEE $802.16 \mathrm{~m}$ ", IEEE, Globecom 2010, pp 1-6

[13] Saran Chiwtanasuntorn, BhumeBhumiratana,EricFiliol,"Perseus on VoIP Development and Implementation of VoIP Platforms", ECTI-CON, IEEE-2014, pp 1-5

[14] Yi Deng, Xiaobo Yu, Qinmin Yang, Jiangang Lu, "On Qos Performance of Different Voice Codecs Over Ad Hoc Networks In Underground Mines", Chinese Control
Conference, IEEE-2014, pp-5521-5526

[15] Zahraa Sabra and Hassan Artail,"Preserving Anonymity and Quality of Service for VoIP Applications over Hybrid Networks", IEEE Mediterranean Electrotechnical Conference, 2014, pp-421-425

[16] Ramon Sanchez-Iborra, Maria-Dolores Cano, Joan Garcia-Haro, "Performance Evaluation of BATMAN Routing Protocol for VoIP Services: A QoE Perspective", Wireless Communications, IEEE, VOL. 13 (9), 2014, pp-4947-4958

[17] Floriano De Rango, Member, IEEE, Peppino Fazio, Student Member, IEEE, Francesco Scarcello, and Francesco Conte,"A New Distributed Application and Network LayerProtocol for VoIP in Mobile Ad Hoc Networks", IEEE TRANSACTIONS ON MOBILE COMPUTING, VOL.13(10), IEEE-2014, pp-2185-2198

[18] http://www.ns2-VoIP.html available at: VoIP Basics: About Jitter 